\title{
Improved Performance of AODV Routing Protocol with Increasing Number of Nodes using Traveling Salesman Problem
}

\author{
Tripti Pandey \\ Shobhit University, \\ $\mathrm{NH}-58$, Modipuram, Meerut
}

\author{
Sangeeta Solanki \\ Shobhit University, \\ $\mathrm{NH}-58$, Modipuram, Meerut
}

\author{
Ramnesh Dubey \\ Nehru Gram Bharti University \\ Jamunipur,Kotwa, Allahabad
}

\begin{abstract}
A mobile ad hoc network is a self-configuring and self organizing infrastructure less network of mobile nodes; these nodes are dynamic in nature and are capable of communicating with each other without the use of a network infrastructure or any centralized administration. With the ease of deployment and the infrastructure less nature of Mobile Ad hoc Networks (MANETs) make them highly popular for the current multimedia communications, so there has been considerable research in routing area. Research shows that Ad Hoc on Demand Distance Vector Routing Protocol (AODV) performs better than any other protocol. Although it performs well but there must be a mechanism to analyze its performance by varying network size. In this paper analyzing the performance of AODV using Travelling Salesman Problem by increasing number of nodes as it is known that routing protocols makes an important task for improving QoS in Mobile Ad hoc Network. The Qos depends upon several parameters like throughput, network load etc. Only throughput parameter has been considered for the simulation. The simulation work has been carried out in Network Simulator (ns-2).
\end{abstract}

\section{KEYWORDS: MANET, AODV and ns-2}

\section{INTRODUCTION}

MANET is usually a self-organizing and self-configuring "multi-hop" network which does not require any fixed infrastructure and where the structure of the network changes dynamically. This change in structure is due to node mobility which also affects the communication between the nodes. So there is a requirement of routing procedure which is always ready to find a path between source and destination to forward the packets appropriately. Routing protocol can be classified as Table driven (Proactive) and On demand (Reactive) routing protocol. In AODV [17] Routes are discovered as on-demand basis and are maintained as long as they are required. It maintains a sequence number, which it increases each time it finds a change in the topology of its neighborhood. This sequence number ensures that the most recent route is selected for execution of the route discovery. AODV is able to provide unicast, multicast and broadcast communication ability. Route tables used by AODV store the destination and next hop IP addresses as well as the destination sequence number. AODV also provide quick deletion of invalid routes in response the route ERROR messages generated due to link breakage. If a node fails to receive three consecutive HELLO messages from a neighbor, it is concluded that link is broken for the specific node and a RERR message is broadcasted to any upstream node. AODV routing protocol seems to be best among all protocols as it gives maximum throughput, lower end to end delay, and higher packet delivery ratio.A problem has been identified what happens when the number of nodes increases gradually this result in packet duplication. So there must be a need to enhance its performance when number of nodes increased. There must be a technique to avoid packet duplication and also a possible way to enhances AODV protocol performances. This paper is organized as follows: In section 2 related work has been there. In section 3 there is a proposed work. Finally conclude in section 4 while section 5 gives the future scope of this work.

\section{RELATED WORK}

In paper [1] it achieves the energy information exchange among neighboring nodes through already-existed signaling packets in AODV and introduces a new network parameter as the comparison threshold, called current average energy of the network, which can fairly accurate estimate the mean power consumption of the cumulative mobile network [1].In paper [39] it uses alternate path FTMR [6] and energy booster mechanism [7] to retransmit the data whenever an intermediate node is unable to continue the process due to node failure or link failure. In this process when a RREQ is received by the node initially, it checks itself for the destination node and also the route to the destination if available in it. Else the entire process will proceed as conventional AOMDV protocol [5]. If the destination node is itself, the first received RREQ is kept in the buffer and simultaneously starts the timer. Other copies of RREQ are also received by the node at the same time. All the copies of RREQ are tested for a new path or node-disjoint. If the RREQ has a new destination or link-disjoint path then the RREQ is kept in the buffer else discarded. Once the RREQ is stored the timer is triggered and when it expires the request is dropped. The node replies with more number of RREPs if it finds low battery power in it. When an upstream node sends the RREP, the number of nodes or hops that the data has travelled is found with the help of the downstream node with the same data. Also the remaining number of nodes or hops to be travelled is calculated mathematically.

Routes in AODV are maintained using periodic hello packets, similar to ackreq packets in DSR. When a critical number of hello packets are sent without a response, newly arrived traffic is stored in a temporary buffer while route repair operations are attempted. If these operations fail, the buffered traffic is discarded, and a RERR is sent to the source node. The critical number of lost hello packets is a user-defined parameter which effectively controls how quickly the network fails broken routes. A small value will result in fast link breakage, and may add unnecessary route discovery traffic during periods of network congestion. In paper [AOMDV [5] protocol focuses on Ad-hoc on demand Multi-Path Distance 
Vector routing challenging AODV in performance. In this researchers analyze the performance of AODV and want to add multipath capability on it [18]. This is very clear we are going to enhance the current AODV functionality to make it multipath. They used NS-3.13 simulator as test bed and enhanced its AODV protocol to work as a multipath routing protocol for achieving better throughput and performance in highly dynamic MANET environment.

\section{PROPOSED WORK}

\subsection{Conventional Ad Hoc On Demand} Distance Vector Routing Protocol (AODV)

In AODV protocol source initiates route to destination when needed. Whenever source node has something to forward it broadcast a Route request packet to the destination node, Route request packet contains the following information: Source address, Broadcast ID, Source sequence number, Destination address, Destination sequence and Hop count. The intermediate nodes receiving this packet checks out its address and if it is not the destination node it broadcast the packet to the other nodes. Node with the destination address sends a Route reply packet to the source node. Once the source node receives the Route reply [17], it may initiate to forward data packets to the destination. To keep track of freshness of routes sequence numbers are used. A route is considered to be active as long as there are data packets periodically travelling from the source to the destination along that path. Once the source stops sending data packets, the links will time out and eventually be deleted from the intermediate node routing tables. If a link break occurs while the route is active, the node upstream of the break propagates a Route error message to the source node to notify it of the now unreachable destination(s). After receiving the Route error message, if the source node still needs the route, it can reinitiate route discovery.

Table 1: Simulation Parameter for AODV

\begin{tabular}{|c|c|}
\hline 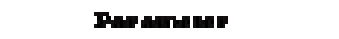 & raher \\
\hline Mhink-17 at nodis & 5 \\
\hline IJnew/andmu & 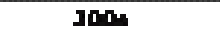 \\
\hline 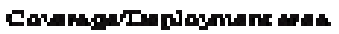 & $\operatorname{lnon}+700 \mathrm{~m}$ \\
\hline hrowak Tya & 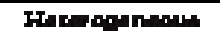 \\
\hline 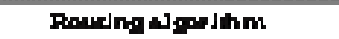 & ADTH \\
\hline & UnHtom Fundam \\
\hline
\end{tabular}

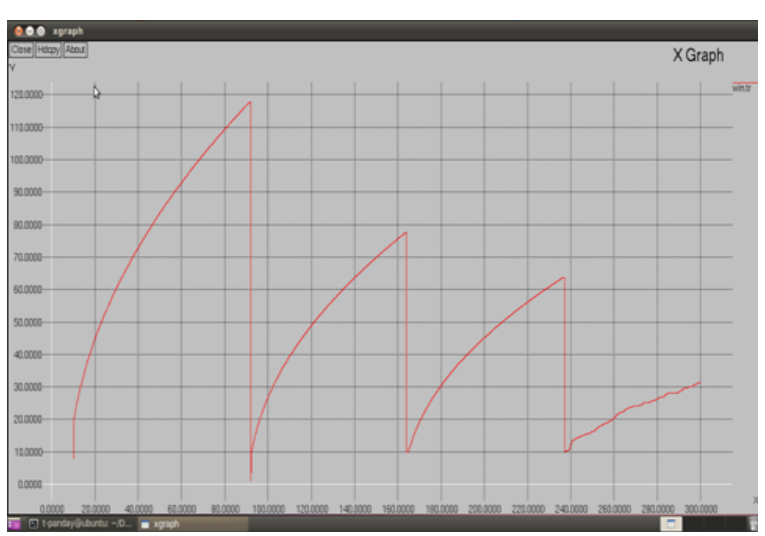

Figure 1:X Graph of AODV Protocol

\subsection{Ad Hoc on Demand Distance Vector Routing Protocol (AODV) with Travelling Salesman Problem}

In our proposed work we are applying Travelling Salesman Problem with Conventional Ad Hoc on Demand Distance Vector Routing Protocol (AODV) in order to minimize network load on the network and to maintain the throughput of the existing protocol, we are analyzing the performance of routing protocol by increasing number of nodes up to 50 nodes we have done our work, as we know routing protocols make an significant task for improving QoS in Mobile Ad hoc Network. Travelling Salesman Problem states that:

If there are $\mathrm{n}$ nodes, and the time between each pair of these nodes is given, find the shortest path where each node is visited exactly once and returning to the source.

In this stated problem number of nodes and time of travelling between the nodes are given we have to determine the possible shortest path from source to destination where each node will be visited only once. The overall algorithm is as follows:

\section{INPUT}

- $\quad$ Number of nodes, $n$

- Time of travelling between the nodes.

- $\quad t(i, j) i, j=1, \ldots, n$

- $\quad$ Start with node 1 (or choose randomly)

\section{OUTPUT}

- Vector of nodes and total time

\section{MAIN STEPS}

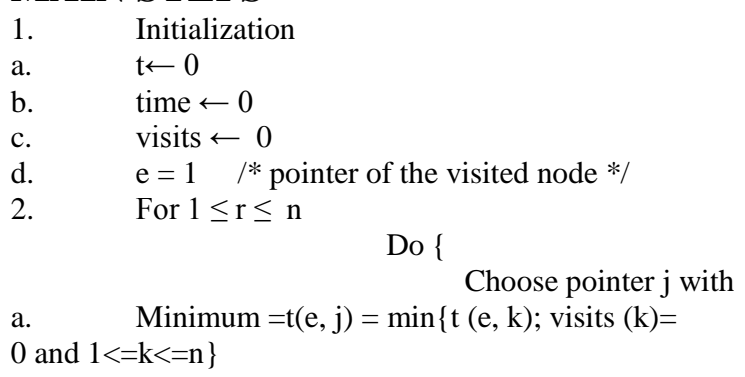

Table 2: Simulation Parameter for implementing TSP with AODV

\begin{tabular}{|c|c|}
\hline Fixuntix & Whum \\
\hline Nhanbur of rodes & ه5. \\
\hline gimalntimtimn & $400 s$ \\
\hline 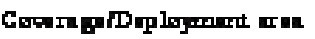 & 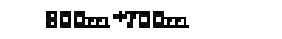 \\
\hline Nutwing Ty" & Hintereprugess \\
\hline Rorting aldprifm & AODV \\
\hline Nad Distr mutim & Urifmm Rmim \\
\hline Pexhart 8 in & 512 bytes \\
\hline PackantRata & 5 purlauts \\
\hline Theffic 8 im & Carstmrt Hil Rntu (CGR) \\
\hline
\end{tabular}




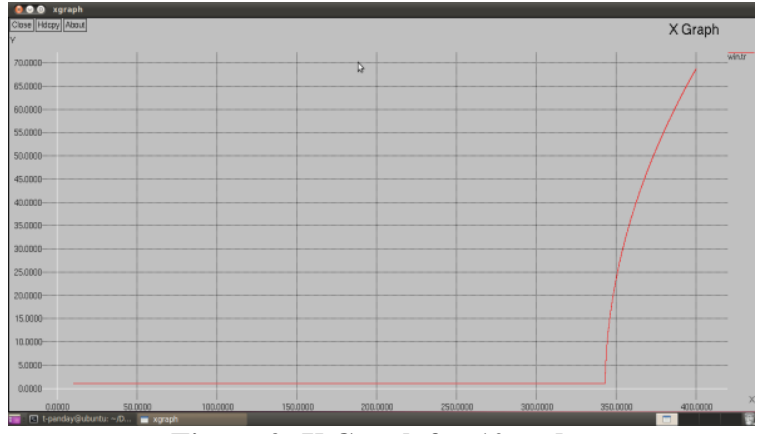

Figure 2: X Graph for 10 nodes

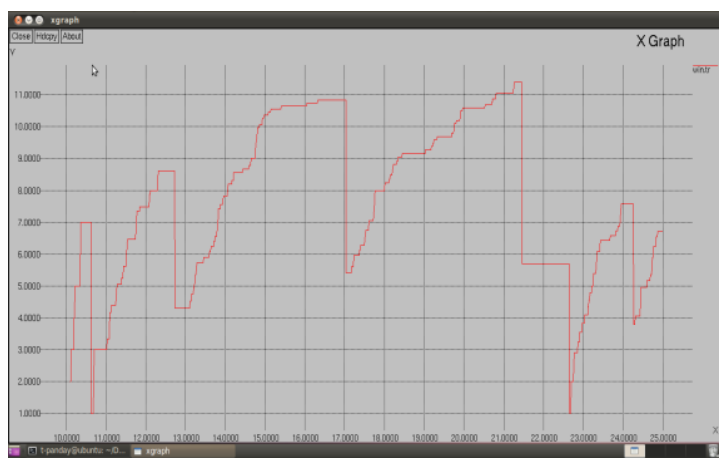

Figure 3: X Graph for 20 nodes

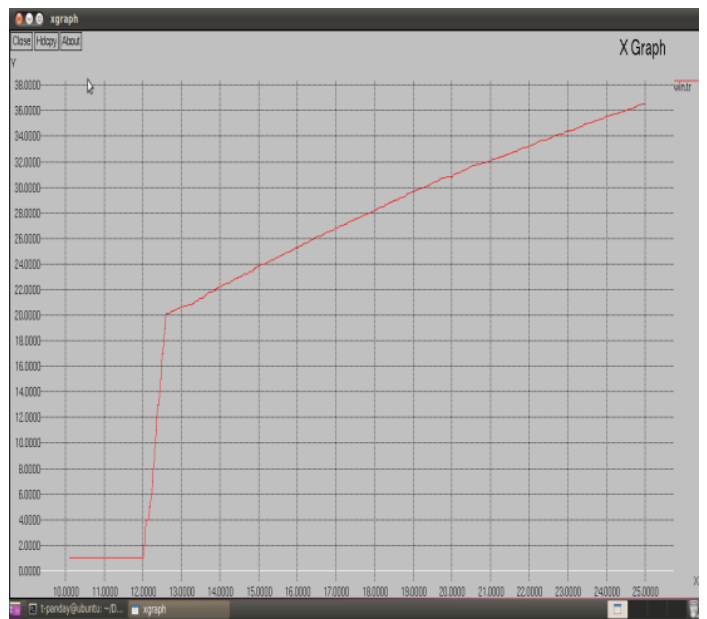

Figure 4: X Graph for 30 nodes

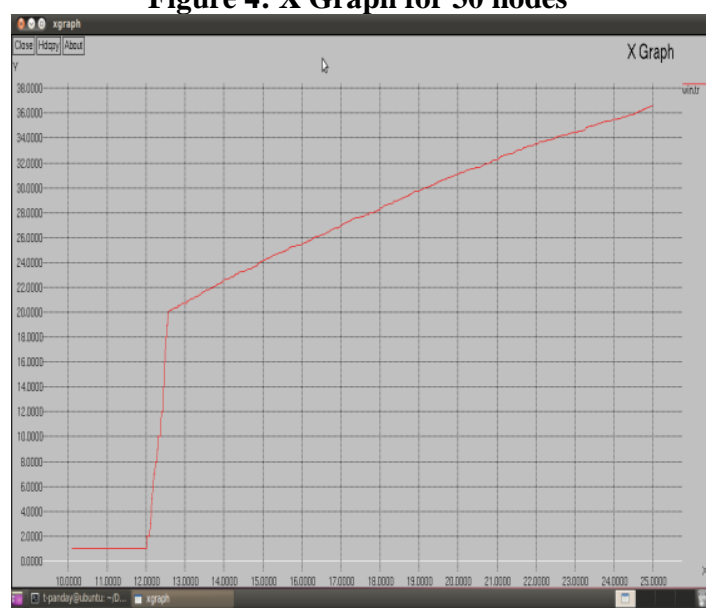

Figure 5: X Graph for 40 nodes

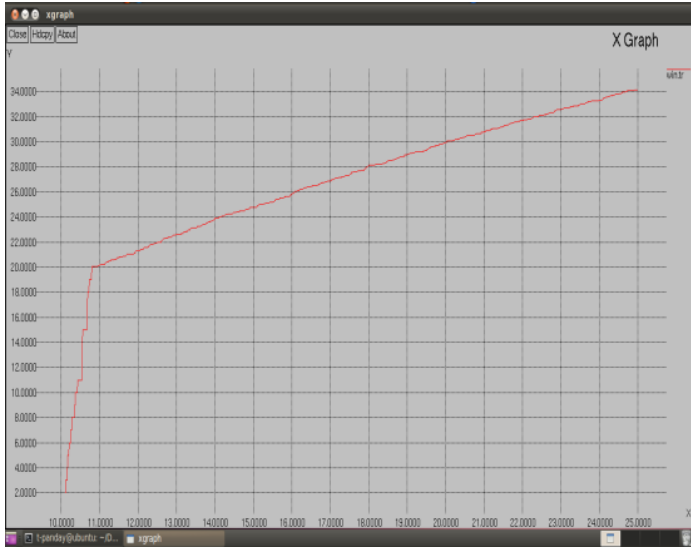

Figure 6: X Graph for 50 nodes
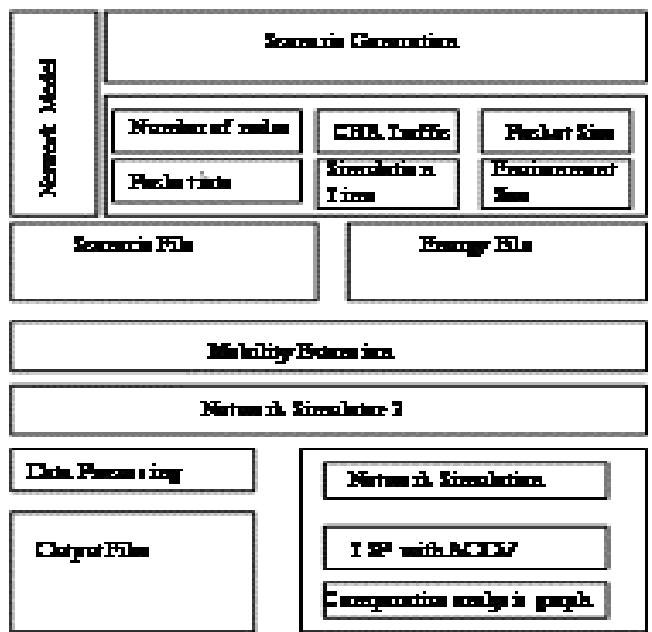

Figure 7: Proposed Architecture

\section{CONCLUSION}

In this proposed work Travelling Salesman Problem with Conventional Ad Hoc on Demand Distance Vector Routing Protocol (AODV) is applied in order to minimize network load on the network and to maintain the throughput of the existing protocol, we are analyzing the performance of routing protocol by increasing number of nodes up to 50 nodes this work has been done, as it is known that routing protocols makes a significant task for improving QoS in Mobile Ad hoc Network.

Simulation is carried out by increasing the number of nodes in order to analyze the performance of proposed routing protocol but time of simulation kept constant i.e. 400 seconds. Simulation for 10 nodes shows that the throughput remains constant for certain period of time but as the time increases throughput also increases. By analyzing all the X Graph it is concluded that the simulation for 50 nodes performs well, here throughput rises at the initial stage and as the time increases throughput also increases and reaches to maximum and it is also observed that packet loss is also minimum in this case while maximum in the case of simulation performed for 30 and 40 nodes.

\section{FUTURE SCOPE}

This work has been carried out by varying network size while the simulation time kept constant. Only throughput parameter is considered in this work, for future work other parameters can be considered such as end to end delay, packet delivery ratio and varying simulation time. It would be interesting to observe the behavior of this proposed protocol by varying 
these network parameters.Network simulator ns-2 is used for simulation, different simulators can also be used such as Qualnet, Opnet, Glomosim etc.

\section{REFERENCES}

[1] Rajesh kumar Yadav, Deepak panwar "Optimal and Robust Framework for Enhancing Network Lifetime using Power Efficient AODV in Mobile Ad hoc Network" Amity University Rajasthan, Jaipur Computer Science \& Engineering.

[2] Hadi Sargolzaey, Ayyoub Akbari Comparison of Reliable unicast routing protocols for mobile ad hoc networks 2009.

[3] Yu-Chee Tseng, Wen-Hua Liao, Shih-Lin Wu, "Mobile Ad Hoc Networks and Routing Protocols", Handbook of Wireless Networks and Mobile Computing, Edited by Ivan Stojmenovic' Copyright (92002 John Wiley \& Sons, Inc. ISBNs: 0-471- 41902-8 (Paper); 0-471- 22456-1 (Electronic).

[4] Srdjan Krco and Marina Dupcinov, "Improved Neighbor Detection Algorithm for AODV Routing Protocol", IEEE Communications Letters, vol. 7, No.12, December 2003.

[5] Mahesh M Karina and Samir R.Das. "Ad hoc On demand multipath distance vector routing”,IEEE 2001

[6] A Fault Tolerant Multipath Routing Protocol to Reduce Route Failures in Mobile Ad Hoc Networks. G. Rajkumar, K.Duraisamy.

[7] A Mechanism For Booster Approach In Mobile Ad Hoc Networks, Ahmad Anzar, Hussain shanawaz, Dr. S.C Gupta.

[8] Elizabeth M. Royer "A Review of Current Routing Protocols for Ad Hoc Mobile Wireless Networks", University of California

[9] Fahim Maan, Nauman Mazhar National University of Sciences and Technology (NUST), MANET Routing
Protocols vs Mobility Models: A Performance Evaluation.

[10] C. E. Perkins, E. M. Belding-Royer, and S. R. Das, "Ad hoc On-Demand Distance Vector (AODV) routing," Internet Engineering Task Force (IETF) draft, November 2002. Available at http://www.ietf.org/internetdrafts/draft-ietf-manet-aodv-12.txt.

[11] Vincent D.Park and M Scott Corson, Temporally Ordered Routing Algorithm (TORA) version 1:Functiona specification , Internet draft, draft ietf manettoraspec-01 .txt August 1998.

[12] V.D Park and M.S.Corson A Highly Adaptive distributed routing algorithm for mobile wireless networks april 1997.

[13] C.-K. Toh, Georgia Institute of Technology Maximum Battery Life Routing to Support Ubiquitous Mobile Computing in Wireless Ad Hoc Networks.

[14] Morteza Maleki, Karthik Dantu, and Massoud Pedram Dept. of EE-Systems, University of Southern California, Los Angeles, Power-aware Source Routing Protocol for Mobile Ad Hoc Networks.

[15] E.M.Royer and Charles E.Perkins multicast operations of the ad hoc on demand distance vector routing algorithm University of California Santa Barbara.

[16] Rohit Dube, Cynthia D.Rais Kuang stability-based adaptive Routing (SSA) for mobile ad hoc networks IEEE Personal Communication.

[17] S.S. Tyagi, R.K. Chauhan Performance Analysis of Proactive and Reactive Routing Protocols for Ad hoc Networks.

[18] Simmi Jain, Prof.Hitesh Gupta, Prof.Mukesh Kumar Baghel Survey on MANET Routing Protocol and proposed Multipath Extension in AODV. 